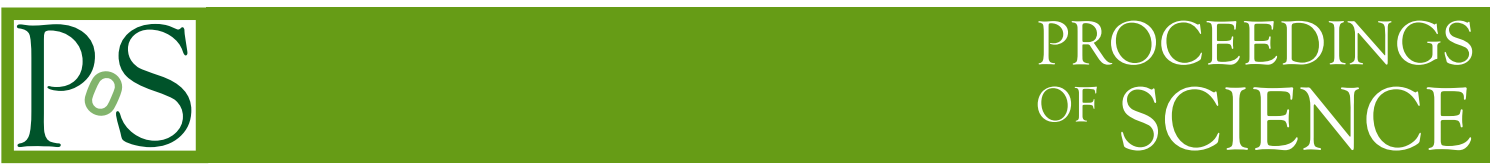

\title{
Hands on DarkSide-50: Low Energy Calibration
}

\section{Kyungwon Kim*}

Seoul National University

IBS Center for Underground Physics

E-mail: kwkimahep1.snu.ac.kr

\section{Benjamin Shanks ${ }^{\dagger}$}

University of North Carolina - Chapel Hill

Triangle Universities Nuclear Laboratory

E-mail: bshanks@unc.edu

\section{Aldo Lanni}

Laboratori Nazionali del Gran Sasso

E-mail: aldo.iannielngs.infn.it

\section{Nicola Rossi}

Laboratori Nazionali del Gran Sasso

E-mail: g.krossi@gmail.com

The DarkSide-50 experiment is a direct detection dark matter search located in Gran Sasso National Laboratory (LNGS). It utilizes a liquid argon time projection chamber to search for weakly interacting massive particles (WIMPS) in the low energy region below $100 \mathrm{keV}$. In order to understand any signals in this region and distinguish WIMP candidate events from background, it is necessary to perform a precise calibration between scintillation light yield and energy deposited in the detector. Presented here are the results of a calibration using the $565 \mathrm{keV}$ endpoint ${ }^{39} \mathrm{Ar}$ beta spectrum together with a $41.5 \mathrm{keV}{ }^{83 \mathrm{~m}} \mathrm{Kr}$ gamma peak. Because the detector is operated under an applied electric fields, additional field- and energy-dependence can affect the calibration in this energy regime. A method to correct for the energy dependence of the light yield is studied and the systematic uncertainty introduced by the fit is analyzed in detail.

Gran Sasso Summer Institute 2014 Hands-On Experimental Underground Physics at LNGS - GSSI14, 22 September - 03 October 2014

INFN - Laboratori Nazionali del Gran Sasso, Assergi, Italy

\footnotetext{
* Speaker.

† Speaker.
} 


\section{Introduction}

\subsection{The DarkSide-50 Experiment}

A preponderance of evidence suggests that the matter component of the universe is dominated by a non-luminous and non-baryonic "dark matter." One proposed dark matter candidate is the early interacting massive particle (WIMP). A number of experiments have been designed to directly detect the low energy $(<100 \mathrm{keV})$ nuclear recoil resulting from the collision of a WIMP within the sensitive volume of a detector.

The best current limits on WIMP cross section have been measured using two-phase liquid xenon time projection chamber (TPC) detectors [1] . The DarkSide-50 design is a two-phase TPC filled with $50 \mathrm{~kg}$ of liquid argon (LAr). Argon can reach extremely low backgrounds by distinguishing electron recoil events from nuclear recoils using pulse shape discrimination (PSD) [2]. However, argon can be cosmogenically activated to long-lived ${ }^{39} \operatorname{Ar}\left(T_{1 / 2}=269 \mathrm{yr}\right)$, which beta decays with a continuous spectrum up to $565 \mathrm{keV}$. To avoid this source of background, physics runs in the DarkSide detector are performed with underground-sourced argon,which has a reduction of ${ }^{39} \mathrm{Ar}$ of $>150$ compared to atmospheric sources. The first results from the DarkSide-50 detector are the best WIMP limits achieved thus far in argon [3].

\subsection{Low Energy Calibration}

In order to calibrate the energy response of prompt scintillation light (S1) in the low energy region, short-lived ${ }^{83 \mathrm{~m}} \mathrm{Kr}$ is injected in LAr of atmospheric origin. The measured S1 spectrum at zero and $0.2 \mathrm{kV} / \mathrm{cm}$ drift field was used in this study to determine the light yield in photoelectrons (PE), and detector performance related to light yield and drift field. In addition to the $41.5 \mathrm{keV}$ gamma peak of ${ }^{83 \mathrm{~m}} \mathrm{Kr}$, the presence of ${ }^{39} \mathrm{Ar}$ allows for a fit to the beta spectrum of ${ }^{39} \mathrm{Ar}(\mathrm{Q}=565$ $\mathrm{keV})$. To account for the detector resolution, the ${ }^{39} \mathrm{Ar}$ spectral function is convolved with a gaussian function, as shown in Eq. 1.1.

$$
F(P E)=G(P E)_{K r}+G(P E)_{A r} * A r 39(P E)
$$

where $\operatorname{Ar} 39(\mathrm{PE})$ is the beta spectrum of ${ }^{39} \mathrm{Ar}$ and $*$ is the convolution operator, and

$$
G(P E)=\frac{1}{\sqrt{2 \pi} \sigma(P E)} \exp \left(\frac{-0.5 P E^{2}}{\sigma^{2}(P E)}\right)
$$

In this study, the convolution was performed via numerical integration of the resolution function with the functional form of the first-forbidden ${ }^{39} \mathrm{Ar}$ beta spectrum function. The variance of detector resolution, sigma(PE), in Eq. 1.2 is described as:

$$
\sigma(P E)=\sqrt{a^{2}+\left(1+b^{2}\right) P E+c^{2} P E^{2}}
$$

where
a: the noise parameter
b: the single PE resolution fixed at 0.4 from laser calibrations
c: the effective Fano parameter 


\section{Analysis}

\subsection{Fit systematics of light yield}

We fit the S1 spectrum with ${ }^{83 \mathrm{~m}} \mathrm{Kr}$ gamma peak and ${ }^{39}$ Ar beta spectrum using function described Eq. 1.1 to determine best light yield at zero drift field. The fit of the entire spectrum at zero drift field is shown in Figure 2.1. ${ }^{83 \mathrm{~m}} \mathrm{Kr}$ peak and ${ }^{39} \mathrm{Ar}$ sepectrum are fitted together with resolution Eq. 1.3 which was set as an independent free parameter in ${ }^{83 \mathrm{~m}} \mathrm{Kr}$ and ${ }^{39} \mathrm{Ar}$. It returns $7.891 \pm$ $0.002 \mathrm{PE} / \mathrm{keV}$ and $7.898 \pm 0.004 \mathrm{PE} / \mathrm{keV}$ of light yield of ${ }^{83 \mathrm{~m}} \mathrm{Kr}$ and ${ }^{39} \mathrm{Ar}$ respectively with the statistical error. The light yield from the fitting ${ }^{83 \mathrm{~m}} \mathrm{Kr}$ and ${ }^{39} \mathrm{Ar}$ spectrum gives consistent results within the statistical error.

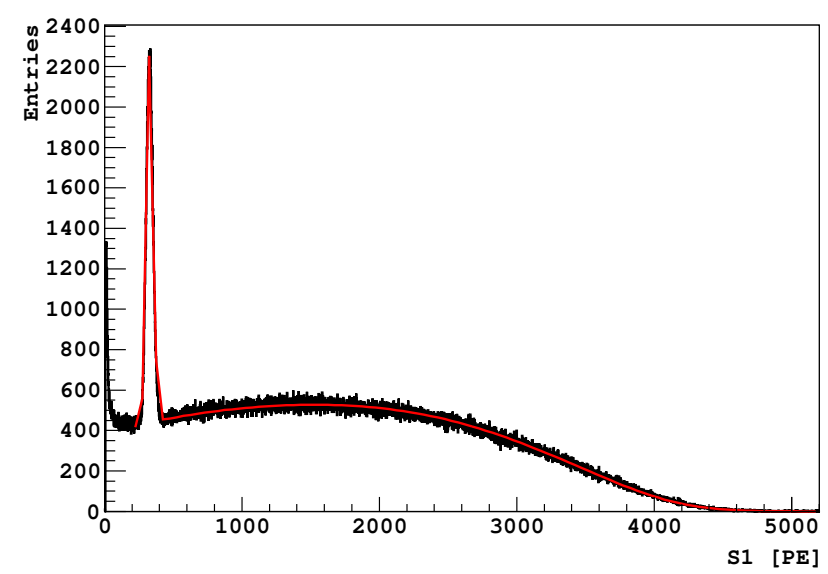

Figure 1: The primary S1 spectrum at zero drift field. Fits are made to the ${ }^{83 \mathrm{~m}} \mathrm{Kr}$ and ${ }^{39} \mathrm{Ar}$ spectra.

The dominant systematic uncertainty in light yield comes from Q-value of ${ }^{39} \mathrm{Ar}$. We fit the ${ }^{39} \mathrm{Ar} \mathrm{S} 1$ spectrum without ${ }^{83 \mathrm{~m}} \mathrm{Kr}$ giving Q-value variation $\pm 1 \%$ of the central, $565 \mathrm{keV}$, to account for systematic uncertainty. Parameter 'a' and 'c' in resolution, Eq. 1.3, was fixed at values from fitting results with the central Q-value. Fit results of Q-value variation are shown in Table 2.1. Q-value variation yields $\pm 1 \%$ systematic uncertainty in the light yield. Consequently, the light yield of ${ }^{39} \mathrm{Ar}$ becomes $7.898 \pm 0.080 \mathrm{PE} / \mathrm{keV}$ with statistical and systematic uncertainty at zero drift field.

\begin{tabular}{lccc} 
& $\mathrm{Q}-1 \%$ & $\mathrm{Q}(565 \mathrm{keV})$ & $\mathrm{Q}+1 \%$ \\
\hline Light Yield (PE/keV) & $7.964 \pm 0.004$ & $7.883 \pm 0.006$ & $7.803 \pm 0.004$ \\
Uncertainty (\%) & $1.030 \pm 0.090$ & & $1.010 \pm 0.090$
\end{tabular}

Table 1: Systematic effect of the Q-value of ${ }^{39} \mathrm{Ar}$

We treat the Q-value as a nuisance parameter in the estimator, chi-square function, in order to estimate systematic uncertainty due to changing Q-value more detaily. Chi-square estimator including nuisance parameter used in the fit is defined as: 


$$
\chi^{2}(L Y, a, c ; \alpha)=\chi^{2}(L Y, a, c)+\left(\frac{\alpha}{h}\right)^{2}
$$

where $a$ and $c$ are parameters in Eq. 1.3, $\alpha$ is parameter of Q-value variation, and $h$ is the error of $\alpha$ in unit of $\%$.

By fitting the ${ }^{39} \mathrm{Ar}$ spectrum with Eq. 1.1 function and Eq. 2.1 estimator, we get the result varying error of the Q-value $0.5 \%$ through $2 \%$ instead of fixing $\alpha$ at 1 as above estimation. The results are given in Table 2.1.

\begin{tabular}{lcccc}
$\mathrm{h}(\%)$ & 0.5 & 0.7 & 1 & 2 \\
\hline$\alpha(\%)$ & $-0.533 \pm 0.528$ & $-1.006 \pm 0.807$ & $-1.908 \pm 1.414$ & $-5.367 \pm 0.288$ \\
Light Yield (PE/keV) & $7.926 \pm 0.341$ & $7.965 \pm 0.066$ & $8.040 \pm 0.118$ & $8.340 \pm 0.026$ \\
Unceratinty of LY(\%) & $-0.547 \pm 0.552$ & $-1.038 \pm 0.847$ & $-1.986 \pm 1.504$ & $-5.794 \pm 0.341$
\end{tabular}

Table 2: Fitting results of Q-value variation parameter

\subsection{Systematic uncertainty from Field effect}

To estimate nuclear recoil energy from measured energy, quenching of nuclear recoil which is measured at $0.2 \mathrm{kV} / \mathrm{cm}$ relative to the light yield of ${ }^{83 \mathrm{~m}} \mathrm{Kr}$ at null field should be considered. Non uniformity of the light collection between top and bottom makes poor resolution, which could be accounted by z-position information from S1 scintillation signal and S2 ionization signal when drift field is on [3]. Since resolution could not be corrected at null field, we estimated systematic uncertainty of resolution.

Fitting ${ }^{83 \mathrm{~m}} \mathrm{Kr}$ and ${ }^{39} \mathrm{Ar} \mathrm{S} 1$ spectrum at zero and $0.2 \mathrm{kV} / \mathrm{cm}$ drift field is carried out with Eq. 1.3 sigma in fit function as fitting range set in ${ }^{83 \mathrm{~m}} \mathrm{Kr}$ gamma peak region. Resolution of ${ }^{83 \mathrm{~m}} \mathrm{Kr}$ gamma peak is $7.260 \pm 0.221 \%$ at zero drift field and $6.399 \pm 0.033 \%$ at $0.2 \mathrm{kV} / \mathrm{cm}$. It returns that systematic uncertainty of resolution is $3.430 \%$ and resolution of ${ }^{83 \mathrm{~m}} \mathrm{Kr}$ is $7.260 \pm 0.333 \%$ with systematic uncertainty.

\subsection{Energy dependence of light yield}

The operation of a dual phase TPC requires the application of a strong electric field which drifts ionization electrons upwards through the chamber. A prompt scintillation signal (S1) is recorded immediately after the ionization, while a delayed scintillation signal (S2) occurs when the ionization electrons are accelerated through the gas-liquid transition surface. Because a portion of the S1 light is generated when ionization electrons recombine with ionized argon, the application of a drift field reduces the mean light yield at a given energy. This effect has been studied in detail in ${ }^{39}$ Ar by the SCENE collaboration [4].

As discussed above, the light yield for zero field was determined to be $7.883 \pm 0.006 \mathrm{PE} / \mathrm{keV}$ using the ${ }^{39} \mathrm{Ar}$ and ${ }^{83 \mathrm{~m}} \mathrm{Kr}$ spectra. Similar data, with only the ${ }^{39} \mathrm{Ar}$ beta spectrum, was taken with an electric field of $0.2 \mathrm{kV} / \mathrm{cm}$ applied. A new fit was performed to this data, floating the light yield parameter as a constant value, but fixing the uncertainty parameters in Eq. 1.3 from the zero field 
fit. With a constant light yield parameter, the fit function poorly replicated the high and low energy regions of the spectrum.

By altering the light yield from a constant parameter to a second degree polynomial of PE, the fit improved dramatically. This fit was robust against floating the uncertainty parameters. Introducing a third degree into the polynomial could not improve on the fit. The results are compiled in Table 2.3 and the resulting fit is shown in Figure 2.3. Over the fit region, from 150 to 3400 PE, the light yield decreases from $6.7 \mathrm{PE} / \mathrm{keV}$ to $5.5 \mathrm{PE} / \mathrm{keV}$.

\begin{tabular}{cc} 
Polynomial Order Constant & Best Fit Value \\
\hline 0 & $6.75 \mathrm{PE} / \mathrm{keV}$ \\
1 & $-3.9 \times 10^{-4} \mathrm{PE} / \mathrm{keV} / \mathrm{PE}$ \\
2 & $4.7 \times 10^{-9} \mathrm{PE} / \mathrm{keV} / \mathrm{PE}^{2}$
\end{tabular}

Table 3: Fitting results with light yield as a second degree polynomial in the region from $150<P E<3400$. The number associated with each constant corresponds to the order of the polynomial constant.

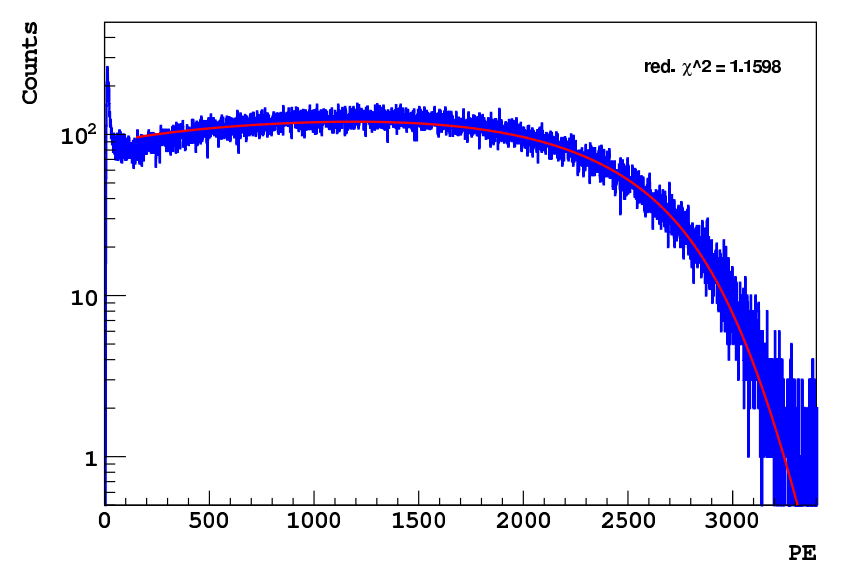

Figure 2: Fit of the ${ }^{39} \mathrm{Ar}$ spectrum allowing the light yield to vary with PE as a second order polynomial in the region from $150<P E<3400$. 


\section{Conclusions}

We studied the light yield of LAr in DarkSide-50 detector. The light yield of ${ }^{39} \mathrm{Ar}$ and ${ }^{83 \mathrm{~m}} \mathrm{Kr}$ are consistent in the statistical error and the dominant systematic uncertainty in light yield comes from ${ }^{39} \mathrm{Ar}$ Q-value which returns about $1 \%$. The systematic uncertainty in resolution of ${ }^{83 \mathrm{~m}} \mathrm{Kr}$ peak energy due to drift field is about $3.430 \%$.

The light yield of the detector was shown to vary strongly with energy. Over the energy range of the ${ }^{39} \mathrm{Ar}$ beta spectrum, the dependence of the light yield on energy can be modeled as a second degree polynomial. The light yield was found to vary by $18 \%$ over the range of the beta spectrum.

\section{References}

[1] D. S. Akerib et al. [LUX Collaboration], Phys. Rev. Lett. 112, no. 9, 091303 (2014) [arXiv:1310.8214 [astro-ph.CO]].

[2] P. Benetti et al. [WARP Collaboration], Astropart. Phys. 49, 495 (2008).

[3] P. Agnes et al. [DarkSide Collaboration], arXiv:1410.0653 [astro-ph.CO].

[4] T. Alexander et al. [SCENE Collaboration], Phys. Rev. D 88, no. 9, 092006 (2013) [arXiv:1306.5675 [physics.ins-det]]. 